\title{
Spectrum of Anxiety Disorders Among Medical Students in a Nigerian Medical School: A Cross-Sectional Study With Standardized Screening Tools
}

\author{
A. T. Chinawa ${ }^{1}$, J. M. Chinawa ${ }^{2}$, E. Aniwada ${ }^{3}$, Oge Amadi $^{4}$, A. C. Ndukuba ${ }^{5} \&$ S. N. Uwaezuoke $^{6}$ \\ ${ }^{1}$ Consultant Community Physician and Lecturer, Enugu State University Teaching Hospital, Enugu State, \\ Nigeria \\ ${ }^{2}$ Senior Lecturer in Pediatrics, College of Medicine, University of Nigeria/University of Nigeria Teaching \\ Hospital (UNTH), Ituku- Ozalla, Enugu State, Nigeria \\ ${ }^{3}$ Lecturer in Community Medicine, College of Medicine, University of Nigeria/University of Nigeria Teaching \\ Hospital (UNTH), Ituku- Ozalla, Enugu State, Nigeria \\ ${ }^{4}$ Consultant Paediatrician, Enugu State University Teaching Hospital, Enugu State, Nigeria \\ 5 Senior Lecturer in Psychological Medicine, College of Medicine, University of Nigeria/University of Nigeria \\ Teaching Hospital (UNTH), Ituku- Ozalla, Enugu State, Nigeria \\ 6. Associate Professor of Pediatrics, College of Medicine, University of Nigeria/University of Nigeria Teaching \\ Hospital (UNTH), Ituku-Ozalla, Enugu State, Nigeria \\ Correspondence: J. M. Chinawa, Senior Lecturer, College of Medicine, Department of pediatrics, University of \\ Nigeria/University of Nigeria Teaching Hospital (UNTH), Ituku- Ozalla, Enugu State, Nigeria. E-mail: \\ josephat.chinawa@unn.edu.ng
}

Received: June 20, 2018 Accepted: August 3, 2018 Online Published: September 3, 2018

doi:10.5539/jedp.v8n2p132 URL: http://doi.org/10.5539/jedp.v8n2p132

\begin{abstract}
Background: Anxiety disorders among medical students constitute a global problem, and also reflect the mental state of the general population. There is paucity of data on the spectrum of such disorders among medical students in Nigeria.
\end{abstract}

Aim: The study aims to determine the prevalence of anxiety disorders among medical students, and the effect of socio-demographic characteristics.

Methods: A total of 217 medical students from the second to the final years of study at Enugu State University of Science and Technology in south-east Nigeria were enrolled by simple random sampling. Five pretested, selfadministered standardized questionnaires were used as screening tools for anxiety disorders. Data were analyzed using the Statistical Package for Social Sciences program (SPSS version 20). A p-value less than 0.05 was taken as statistically significant.

Results: Thirty one (14.3\%) of the enrolled medical students fulfilled the screening criteria for anxiety disorders. Specifically, generalized anxiety disorder (GAD) was significantly related to gender $(p=0.017)$ and the year of study $(p=0.017)$. Post-traumatic stress disorder (PTSD) was significantly related to the year of study $(p=0.037)$, and social anxiety disorder $(\mathrm{SAD})$ to the year of study $(\mathrm{p}=0.003)$ and gender $(\mathrm{p}=0.04)$. Similarly, panic disorder was significantly related to the year of study $(\mathrm{p}=0.025)$ while specific phobia was significantly associated with marital status $(p=0.003)$, parental monthly income $(p=0.022)$ and student's monthly allowance $(p=0.002)$. Finally, obsessive-compulsive disorder was significantly related to marital status $(p=0.034)$ and year of study $(p=0.028)$.

Conclusion: Medical students in Nigeria are prone to a spectrum of anxiety disorders. This susceptibility is influenced by socio-demographic characteristics.

Keywords: anxiety disorders, medical students, standardized questionnaires, Nigeria

\section{Introduction}

The medical student is constantly at risk of physical and psychological stress given the rigorous training programs and didactic syllabus (Bostanci et al., 2005). Constant perturbation of this individual with stressors may result in 
anxiety disorders. Short-term and long-term sequelae of these disorders include additional stress and anxiety, worsening academic performance, psychological or emotional impairment during professional life (which may affect quality of patient care), as well as depression and substance abuse (Gill \& Mohammed, 2010).

Anxiety disorders among medical students remain a global challenge, which also reflect the mental state of the general population (Bostanci et al., 2005; Wittchen, 2002). Reports indicate that these disorders are particularly observed in the students' first academic year (Eller et al., 2006; Wittchen, 2002), and are attributed to academic stressors stemming from curriculum overload, paucity of leisure time and uncertainties which arise from the transit time between secondary school and first year in the university (Eller et al., 2006; Wittchen, 2002).

The burden of anxiety disorders among students is well documented in studies conducted outside Africa (Wittchen, 2002; Goodwin et al., 2005; Kessler et al., 2005; Leon et al., 1995; Asaad \& Aqeel, 2010; Connor et al., 2001) and consists of generalized anxiety disorders (GAD), panic disorders, social anxiety disorders (SAD), phobias, post-traumatic stress disorders (PTSD) and obsessive compulsive disorders (OCDs): with reported prevalence rates of about $35 \%, 2.7 \%, 8 \%, 9.6 \%, 4.7 \%$, and $1.3 \%$ respectively. Notably, these disorders also show a female preponderance.

SAD normally occur during communication skills-based examinations or workshops such as viva voce and clinical presentation (Laidlaw, 2009). PTSD develop following a stressful event of an exceptionally threatening or catastrophic nature (Merikangas, 2004). For medical students, PTSD usually occur after failing a medical examination or when withdrawn from the school after examination failure. In addition, they are prone to developing depression with the attendant consequences to their emotional, mental and physical well-being (Tabalipa et al., 2018).

There is paucity of data on the spectrum of anxiety disorders among medical students in Nigeria. Thus, the present study aims to determine the prevalence of anxiety disorders among these students in this clime, and the effect of socio-demographic characteristics.

\section{Methods}

\subsection{Study Design}

This was a cross-sectional study which assessed the spectrum of anxiety disorders among medical students who attended Enugu State University of Technology Teaching Hospital in Enugu metropolis, south-east Nigeria. The study was conducted during the examination-free period in the University to minimize the effect of bias as much as possible.

\subsection{Study Population}

The study was carried out among medical students from second to the final year of academic study who gave their consent after explaining the optional nature of their participation and the liberty to withdraw from the study at any point. The first-year students (who were off-campus) and other students who failed to give consent were excluded. Questionnaires were administered to these students from Jan 2016 to July 2016. Socioeconomic class was assigned to the students using a method modified by Oyedeji (1985).

\subsection{Questionnaire-Based Screening Tools}

Five questionnaires were used to screen for the spectrum of anxiety disorders among these students. For SAD, the National Institute for Health and Care Excellence (NICE) recommends the use of the Mini-Social Phobia Inventory (Mini-SPIN) (Connor et al., 2001). PTSD was assessed using the 4-item Mini-SPIN which comprise four major questions with a dichotomous 'Yes' or 'No' answer. A score of 'YES' was scored 1 while a score of 'NO' was scored zero. A total score of 2 and above suggests likelihood of having PTSD (The Primary Care PTSD screen, 2016). Panic disorder was assessed with the standardized screening questionnaire which contains 8 major questions with 27 items with a dichotomous answer of 'YES' or 'NO'. An answer of 'YES' was scored 1 while a response of 'NO' was scored zero. A total score of 14 and above suggests panic anxiety disorders (The Primary Care PTSD screen, 2016). Phobia- screening questionnaire was used for specific phobias. It contains 8 items with a dichotomous 'YES' or 'NO' answer. A response of 'YES' was scored 1 while a response of 'NO' was scored zero. A total score of 4 and above suggest phobia (The Primary Care PTSD screen, 2016), OCDs questionnaire contains six items with a dichotomous 'YES' or 'NO' answer. A score of 'YES' was scored 1 while a score of 'NO' was scored zero. A total score of 3 and above suggest phobia (Tabalipa et al., 2018). For GAD, the GAD-scale was used to screen the students (Bibi \& Nasir Ali, 2015). The initial item pool consisted of 8 items that reflected all of the Diagnostic and Statistical Manual of Mental Disorders, Fourth Edition (DSM-IV). The questionnaire was developed by asking the study subjects the frequency by which they were bothered by each symptom during the 
last 2 weeks. Response options were 'not at all', 'several days,' 'more than half the days,' and 'nearly every day': which scored as $0,1,2$, and 3 respectively. In addition, an item to assess duration of anxiety symptoms was included. A score of 52-100\% indicated anxiety disorder or a score of $\geq 11$ on GAD-7 indicated anxiety (Robert et al., 2006).

\subsection{Data Analysis}

Data were analyzed using the Statistical Package for Social Sciences program (SPSS version 20 Chicago.). Chi-square was used to test significant association for the qualitative variables while multivariate logistic regression was used to determine correlation. A p-value less than 0.05 was taken as significant for each statistical test

\subsection{Ethical Consideration}

Ethical clearance was sought and obtained from the Ethics Committee of the Enugu State University Teaching Hospital, Enugu.

\section{Results}

Of the 217 students studied, 120 (55.2\%) were female while 97 (45.8\%) were male, giving a male: female ratio of 1:1.2 Majority of them were aged 21-25 years, while with respect to marital status, $85.3 \%$ were single, while $33.3 \%$ had monthly income of between 10-20 thousand Nigerian Naira (equivalent to 30-60 US dollars). (Table 1).

As shown in Table 2, 31/217 (14.3\%) fulfilled the criteria for anxiety disorder. Furthermore, 86/217 (39.6\%) met the criteria for GAD, 70/217 (32.3\%) for PTSD, 37/217 (17.1\%) for SAD, 11/217 (5.1\%) for panic disorder, 73/217 (33.6\%) for specific phobia and 39/217 (18.0\%) for OCDs.

In Table 3, GAD was significantly related to gender $(\chi 2=5.706, p=0.017)$, year of study $(\chi 2=12.040, p=0.017)$, but not related to age $\left(\chi^{2}=7.413, \mathrm{p}=0.060\right)$, marital status $(\chi 2=3.359, \mathrm{p}=0.067)$, religion $\left(\chi^{2}=0.454, \mathrm{p}=0.500\right)$, parents relationship $(\chi 2=1.901, \mathrm{p}=0.168)$, good personal interaction with parents $(\chi 2=2.030, \mathrm{p}=0.154)$, and use of tobacco $\left(\chi^{2}=1.679, p=0.432\right)$. Similarly, PTSD was significantly related to the year of study $(\chi 2=10.237, p$ $=0.037)$ but not to age $\left(\chi^{2}=5.568, \mathrm{p}=0.135\right)$, marital status $\left(\chi^{2}=2.268, \mathrm{p}=0.122\right)$, religion $(\chi 2=1.222, \mathrm{p}=0.269)$, parents relationship $(\chi 2=0.024, p=0.877)$, good personal interaction with parents $\left(\chi^{2}=1.274, p=0.259\right)$, and use of tobacco $\left(\chi^{2}=0.757, \mathrm{p}=0.685\right)$ ( Table 4$)$.

As displayed in Table 5, SAD was significantly related to the year of study $(\chi 2=15.699, \mathrm{p}=0.003)$ and gender $\left(\chi^{2}\right.$ $=4.044, \mathrm{p}=0.04)$ but not to similar variables like age $(\chi 2=7.534, \mathrm{p}=0.057)$, marital status $(\chi 2=0.550, \mathrm{p}=0.458)$, religion $(\chi 2=0.856, p=0.355)$, parents relationship $(\chi 2=1.979, \mathrm{p}=0.160)$, good personal interaction with parents $\left(\chi^{2}=0.251, \mathrm{p}=0.616\right)$, and use of tobacco $\left(\chi^{2}=5.910, \mathrm{p}=0.052\right)$.

Interestingly, other forms of anxiety disorders showed a similar pattern of relationship with the tested variables. For instance, panic disorder (Table 6$)$ and OCDs (Table 8$)$ were significantly related to the year of study: $(\chi 2=$ $11.189, \mathrm{p}=0.025)$ and $(\chi 2=10.918, \mathrm{p}=0.028)$ respectively. Specific phobia was significantly associated with marital status $(\chi 2=8.595, \mathrm{p}=0.003)$ parents' monthly income $(\chi 2=7.603, \mathrm{p}=0.022)$ and monthly allowance $(\chi 2=$ $14.929, \mathrm{p}=0.002)$ as shown in Table 7 . The variables not significantly related to specific phobia were age $(\chi 2=$ $4.747, p=0.191)$, religion $(\chi 2=0.970, p=0.325)$, parents relationship $(\chi 2=0.082, p=0.775)$, good personal interaction with parents $\left(\chi^{2}=0.276, \mathrm{p}=0.599\right)$, and use of tobacco $\left(\chi^{2}=3.486, \mathrm{p}=0.175\right)$. However, for panic disorder, there was no significant relationship with age $\left(\chi^{2}=4.240, \mathrm{p}=0.237\right)$, marital status $\left(\chi^{2}=2.004, \mathrm{p}=0.157\right)$, religion $\left(\chi^{2}=0.738, \mathrm{p}=0.390\right)$, parents relationship $\left(\chi^{2}=0.182, \mathrm{p}=0.669\right)$ and good personal interaction with parents $(\chi 2=1.787, \mathrm{p}=0.181)$. OCDs were also significantly associated with marital status $(\chi 2=4.489, \mathrm{p}=0.034)$ but not with age $\left(\chi^{2}=7.574, p=0.056\right)$, religion $\left(\chi^{2}=0.244, p=0.621\right)$, parents relationship $\left(\chi^{2}=0.236, p=0.627\right)$, good personal interaction with parents $\left(\chi^{2}=0.863, \mathrm{p}=0.353\right)$, and use of tobacco $(\chi 2=3.925, \mathrm{p}=0.141)$. 
Table 1. Socio-demographic characteristics of respondents

\begin{tabular}{|c|c|c|}
\hline Variables & Frequency $\mathbf{n}=217$ & Percentage \\
\hline \multicolumn{3}{|l|}{ Age(years) } \\
\hline$\leq 20$ & 50 & .23 .0 \\
\hline $21-25 y r s$ & 104 & 47.9 \\
\hline $26-30 y r s$ & 47 & 21.7 \\
\hline Above $30 y r s$ & 16 & 7.4 \\
\hline \multicolumn{3}{|l|}{ Sex } \\
\hline Male & 97 & 44.7 \\
\hline Female & 120 & 55.3 \\
\hline \multicolumn{3}{|l|}{ Marital status } \\
\hline Single & 185 & 85.3 \\
\hline Others (Married, Divorced, widow) & 32 & 14.7 \\
\hline \multicolumn{3}{|l|}{ Tribe } \\
\hline Igbo & 189 & 87.1 \\
\hline Others (Hausa, Yoruba) & 28 & 12.9 \\
\hline \multicolumn{3}{|l|}{ Religion } \\
\hline Christianity & 204 & 94.0 \\
\hline Others (Muslim, traditional) & 13 & 6.0 \\
\hline \multicolumn{3}{|l|}{ Year of study } \\
\hline 200 level & 58 & 26.7 \\
\hline 300 level & 24 & 11.1 \\
\hline 400 level & 70 & 32.3 \\
\hline 500 level & 55 & 25.3 \\
\hline 600 level & 10 & 4.6 \\
\hline \multicolumn{3}{|l|}{ Parent's relationship } \\
\hline Live together & 166 & 76.5 \\
\hline Others(single, separated, widow) & 51 & 23.5 \\
\hline \multicolumn{3}{|l|}{ Your parent's monthly income $n=187$} \\
\hline$\leq 100$ & 57 & 30.5 \\
\hline $101-200$ & 48 & 25.7 \\
\hline$>200$ & 82 & 43.9 \\
\hline \multicolumn{3}{|l|}{ Your monthly allowance ranges } \\
\hline $1-10 \mathrm{k}$ & 63 & 30.9 \\
\hline $10-20 \mathrm{k}$ & 72 & 35.3 \\
\hline $21-30 \mathrm{k}$ & 42 & 20.6 \\
\hline $31-40 \mathrm{k}$ & 27 & 13.2 \\
\hline \multicolumn{3}{|c|}{ Good personal interaction with your parents } \\
\hline Yes & 188 & 86.6 \\
\hline No & 29 & 13.4 \\
\hline \multicolumn{3}{|l|}{ Use of tobacco } \\
\hline Use of tobacco & 13 & 8.5 \\
\hline Use of alcohol & 51 & 33.3 \\
\hline Use of caffeinated drinks/beverages & 89 & 58.2 \\
\hline
\end{tabular}


Table 2. Associations between factors and anxiety disorder (AGGREGATE)

\begin{tabular}{|c|c|c|c|c|}
\hline Variables & Anxiety n(\%) & No anxiety n(\%) & Test statistic $\chi^{2}$ & p value \\
\hline \multicolumn{5}{|l|}{ Age(years) } \\
\hline$\leq 20$ & $17(34.0)$ & $33(66.0)$ & \multirow{4}{*}{1.146} & \multirow{4}{*}{0.766} \\
\hline $21-25 y r s$ & $40(38.5)$ & $64(61.5)$ & & \\
\hline $26-30 y r s$ & $14(29.8)$ & $33(70.2)$ & & \\
\hline Above $30 y r s$ & $6(37.5)$ & $10(62.5)$ & & \\
\hline \multicolumn{5}{|l|}{ Sex } \\
\hline Male & $29(29.9)$ & $68(70.1)$ & \multirow{2}{*}{2.392} & \multirow{2}{*}{0.122} \\
\hline Female & $48(40.0)$ & $72(60.0)$ & & \\
\hline \multicolumn{5}{|l|}{ Marital status } \\
\hline Single & $62(33.5)$ & $123(66.5)$ & \multirow{2}{*}{2.128} & \multirow{2}{*}{0.145} \\
\hline Others (Married, Divorced, widow) & $15(46.9)$ & $17(53.1)$ & & \\
\hline \multicolumn{5}{|l|}{ Tribe } \\
\hline Igbo & $65(34.4)$ & $124(65.6)$ & \multirow{2}{*}{0.763} & \multirow{2}{*}{0.382} \\
\hline Others (Hausa, Yoruba) & $12(42.9)$ & $.16(57.1)$ & & \\
\hline \multicolumn{5}{|l|}{ Religion } \\
\hline Christianity & $71(34.8)$ & $133(65.2)$ & \multirow{2}{*}{0.688} & \multirow{2}{*}{0.407} \\
\hline Others (Muslim, traditional) & $6(46.2)$ & $.7(53.8)$ & & \\
\hline \multicolumn{5}{|l|}{ Year of study } \\
\hline 200 level & $25(43.1)$ & $33(56.9)$ & \multirow{5}{*}{5.311} & \multirow{5}{*}{0.257} \\
\hline 300 level & $11(45.8)$ & $13(54.2)$ & & \\
\hline 400 level & $23(32.9)$ & $47(67.1)$ & & \\
\hline 500 level & $14(25.5)$ & $41(74.5)$ & & \\
\hline 600 level & $4(40.0)$ & $6(60.0)$ & & \\
\hline \multicolumn{5}{|l|}{ Parent's relationship } \\
\hline Live together & $54(32.5)$ & $112(67.5)$ & \multirow{2}{*}{2.692} & \multirow{2}{*}{0.101} \\
\hline Others(single, separated, widow) & $23(45.1)$ & $28(54.9)$ & & \\
\hline \multicolumn{5}{|l|}{ Your parent's monthly income } \\
\hline$\leq 100$ & $20(35.1)$ & $37(64.9)$ & \multirow{3}{*}{3.121} & \multirow{3}{*}{0.210} \\
\hline $101-200$ & $22(45.8)$ & $26(54.2)$ & & \\
\hline$>200$ & $25(30.5)$ & $57(69.5)$ & & \\
\hline \multicolumn{5}{|l|}{ Your monthly allowance ranges } \\
\hline $1-10 \mathrm{k}$ & $22(34.9)$ & $41(65.1)$ & & \\
\hline $10-20 \mathrm{k}$ & $33(45.8)$ & $39(54.2)$ & 5717 & 0126 \\
\hline $21-30 k$ & $13(31.0)$ & $29(69.0)$ & 5.111 & \\
\hline $31-40 \mathrm{k}$ & $6(22.2)$ & $21(77.8)$ & & \\
\hline Good personal interaction with yo & & & & \\
\hline Yes & $64(34.0)$ & $124(66.0)$ & 1277 & 0250 \\
\hline No & $13(44.8)$ & $16(55.2)$ & 1.211 & 0.259 \\
\hline Use of tobacco & & & & \\
\hline Use of tobacco & $4(30.8)$ & $9(69.2)$ & & \\
\hline Use of alcohol & $14(27.5)$ & $37(72.5)$ & 3.972 & 0.137 \\
\hline Use of caffeinated drinks/beverages & $39(43.8)$ & $50(56.2)$ & & \\
\hline
\end{tabular}


Table 3. Associations between factors and General Anxiety Disorder

\begin{tabular}{|c|c|c|c|c|}
\hline Variables & Anxiety n (\%) & No anxiety n (\%) & Test statistic $\chi^{2}$ & p value \\
\hline \multicolumn{5}{|l|}{ Age(years) } \\
\hline$\leq 20$ & $12(24.0)$ & $38(76.0)$ & \multirow{4}{*}{7.413} & \multirow{4}{*}{0.060} \\
\hline $21-25 y r s$ & $45(43.3)$ & $59(56.7)$ & & \\
\hline $26-30 y r s$ & $23(48.9)$ & $24(51.1)$ & & \\
\hline Above $30 y r s$ & $6(37.5)$ & $10(62.5)$ & & \\
\hline \multicolumn{5}{|l|}{ Sex } \\
\hline Male & $47(42.2)$ & $50(51.5)$ & \multirow{2}{*}{5.706} & \multirow{2}{*}{0.017} \\
\hline Female & $39(32.5)$ & $81(67.5)$ & & \\
\hline \multicolumn{5}{|l|}{ Marital status } \\
\hline Single & $78(33.5)$ & $107(57.8)$ & \multirow{2}{*}{3.359} & \multirow{2}{*}{0.067} \\
\hline Others (Married, Divorced, widow) & $8(25.0)$ & $24(75.0)$ & & \\
\hline \multicolumn{5}{|l|}{ Tribe } \\
\hline Igbo & $77(40.7)$ & $112(59.3)$ & \multirow{2}{*}{0.754} & \multirow{2}{*}{0.385} \\
\hline Others (Hausa, Yoruba) & $9(32.1)$ & $19(67.9)$ & & \\
\hline \multicolumn{5}{|l|}{ Religion } \\
\hline Christianity & $82(40.2)$ & $122(59.8)$ & \multirow{2}{*}{0.454} & \multirow{2}{*}{0.500} \\
\hline Others (Muslim, traditional) & $4(30.8)$ & $9(69.2)$ & & \\
\hline \multicolumn{5}{|l|}{ Year of study } \\
\hline 200 level & $13(22.4)$ & $45(77.6)$ & \multirow{5}{*}{12.040} & \multirow{5}{*}{0.017} \\
\hline 300 level & $8(33.3)$ & $16(66.7)$ & & \\
\hline 400 level & $32(45.7)$ & $38(54.3)$ & & \\
\hline 500 level & $28(50.9)$ & $27(49.1)$ & & \\
\hline 600 level & $5(50.0)$ & $5(50.0)$ & & \\
\hline \multicolumn{5}{|l|}{ Parent's relationship } \\
\hline Live together & $70(42.2)$ & $96(57.8)$ & \multirow{2}{*}{1.901} & \multirow{2}{*}{0.168} \\
\hline Others(single, separated, widow) & $16(31.4)$ & $35(68.6)$ & & \\
\hline \multicolumn{5}{|l|}{ Your parent's monthly income } \\
\hline$\leq 100$ & $22(38.6)$ & $35(61.4)$ & \multirow{3}{*}{4.161} & \multirow{3}{*}{0.125} \\
\hline $101-200$ & $13(27.1)$ & $35(79.2)$ & & \\
\hline$>200$ & $37(45.1)$ & $45(54.9)$ & & \\
\hline \multicolumn{5}{|l|}{ Your monthly allowance ranges } \\
\hline $1-10 \mathrm{k}$ & $20(31.7)$ & $43(68.3)$ & & \\
\hline $10-20 \mathrm{k}$ & $25(34.7)$ & $47(65.3)$ & 5309 & 0150 \\
\hline $21-30 \mathrm{k}$ & $18(42.9)$ & $24(57.1)$ & & \\
\hline $31-40 \mathrm{k}$ & $15(55.6)$ & $12(44.4)$ & & \\
\hline Good personal interaction with yo & & & & \\
\hline Yes & $78(41.5)$ & $110(58.5)$ & 2030 & 0154 \\
\hline No & $8(27.6)$ & $21(72.4)$ & 2.050 & 0.134 \\
\hline Use of tobacco & & & & \\
\hline Use of tobacco & $5(38.5)$ & $8(61.5)$ & & \\
\hline Use of alcohol & $24(47.1)$ & $27(52.9)$ & 1.679 & 0.432 \\
\hline Use of caffeinated drinks/beverages & $32(36.0)$ & $57(64.0)$ & & \\
\hline
\end{tabular}


Table 4. Associations between factors and Post-traumatic stress Disorder

\begin{tabular}{|c|c|c|c|c|}
\hline Variables & Presence n (\%) & Absence n (\%) & Test statistic $\chi^{2}$ & p value \\
\hline \multicolumn{5}{|l|}{ Age(years) } \\
\hline$\leq 20$ & $20(40.0)$ & $30(60.0)$ & \multirow{4}{*}{5.568} & \multirow{4}{*}{0.135} \\
\hline $21-25 y r s$ & $30(28.8)$ & $74(71.2)$ & & \\
\hline $26-30 y r s$ & $18(38.3)$ & $29(61.7)$ & & \\
\hline Above $30 y r s$ & $2(12.5)$ & $14(87.5)$ & & \\
\hline \multicolumn{5}{|l|}{ Sex } \\
\hline Male & $25(25.8)$ & $72(74.2)$ & \multirow{2}{*}{2.392} & \multirow{2}{*}{0.122} \\
\hline Female & $45(37.5)$ & $75(62.5)$ & & \\
\hline \multicolumn{5}{|l|}{ Marital status } \\
\hline Single & $56(30.3)$ & $129(69.7)$ & \multirow{2}{*}{2.268} & \multirow{2}{*}{0.132} \\
\hline Others (Married, Divorced, widow) & $14(43.8)$ & $18(56.3)$ & & \\
\hline \multicolumn{5}{|l|}{ Tribe } \\
\hline Igbo & $59(31.2)$ & $130(68.8)$ & \multirow{2}{*}{0.727} & \multirow{2}{*}{0.394} \\
\hline Others (Hausa, Yoruba) & $11(39.3)$ & $.17(60.7)$ & & \\
\hline \multicolumn{5}{|l|}{ Religion } \\
\hline Christianity & $64(31.4)$ & $140(68.6)$ & \multirow{2}{*}{1.222} & \multirow{2}{*}{0.269} \\
\hline Others (Muslim, traditional) & $6(46.2)$ & $.7(53.8)$ & & \\
\hline \multicolumn{5}{|l|}{ Year of study } \\
\hline 200 level & $26(44.8)$ & $32(55.2)$ & \multirow{5}{*}{10.237} & \multirow{5}{*}{0.037} \\
\hline 300 level & $11(45.8)$ & $13(54.2)$ & & \\
\hline 400 level & $16(22.9)$ & $54(77.1)$ & & \\
\hline 500 level & $14(25.5)$ & $41(74.5)$ & & \\
\hline 600 level & $3(30.0)$ & $7(70.0)$ & & \\
\hline \multicolumn{5}{|l|}{ Parent's relationship } \\
\hline Live together & $54(32.5)$ & $112(67.5)$ & \multirow{2}{*}{0.024} & \multirow{2}{*}{0.877} \\
\hline Others(single, separated, widow) & $16(31.4)$ & $35(68.6)$ & & \\
\hline \multicolumn{5}{|l|}{ Your parent's monthly income } \\
\hline$\leq 100$ & $19(33.3)$ & $38(66.7)$ & \multirow{3}{*}{1.398} & \multirow{3}{*}{0.497} \\
\hline $101-200$ & $20(41.7)$ & $28(58.3)$ & & \\
\hline$>200$ & $26(31.7)$ & $56(68.3)$ & & \\
\hline \multicolumn{5}{|l|}{ Your monthly allowance ranges } \\
\hline $1-10 \mathrm{k}$ & $23(36.5)$ & $40(63.5)$ & & \\
\hline $10-20 \mathrm{k}$ & $26(36.1)$ & $46(63.9)$ & 142 & $0=10$ \\
\hline $21-30 \mathrm{k}$ & $13(31.0)$ & $29(69.0)$ & 2.143 & 0.543 \\
\hline $31-40 \mathrm{k}$ & $6(22.2)$ & $21(77.8)$ & & \\
\hline Good personal interaction with yo & & & & \\
\hline Yes & $58(30.9)$ & $130(69.1)$ & 1274 & 0250 \\
\hline No & $12(41.4)$ & $17(58.6)$ & 1.214 & 0.239 \\
\hline Use of tobacco & & & & \\
\hline Use of tobacco & $3(23.1)$ & $10(76.9)$ & & \\
\hline Use of alcohol & $18(35.3)$ & $33(64.7)$ & 0.757 & 0.685 \\
\hline Use of caffeinated drinks/beverages & $31(34.8)$ & $58(65.2)$ & & \\
\hline
\end{tabular}


Table 5. Associations between factors and Social Panic Anxiety Disorder

\begin{tabular}{|c|c|c|c|c|}
\hline Variables & Anxiety n (\%) & No anxiety $n(\%)$ & Test statistic $\left(\chi^{2}\right)$ & p value \\
\hline \multicolumn{5}{|l|}{ Age(years) } \\
\hline$\leq 20$ & $12(24.0)$ & $38(76.0)$ & \multirow{4}{*}{7.534} & \multirow{4}{*}{0.057} \\
\hline $21-25 y r s$ & $21(20.2)$ & $83(79.8)$ & & \\
\hline $26-30 \mathrm{yrs}$ & $3(6.4)$ & $44(93.6)$ & & \\
\hline Above $30 \mathrm{yrs}$ & $1(6.3)$ & $15(93.8)$ & & \\
\hline \multicolumn{5}{|l|}{ Sex } \\
\hline Male & $11(11.3)$ & $86(88.7)$ & \multirow{2}{*}{4.044} & \multirow{2}{*}{0.044} \\
\hline Female & $26(21.7)$ & $94(78.3)$ & & \\
\hline \multicolumn{5}{|l|}{ Marital status } \\
\hline Single & $33(17.8)$ & $152(82.2)$ & \multirow{2}{*}{0.550} & \multirow{2}{*}{0.458} \\
\hline Others (Married, Divorced, widow) & $4(12.5)$ & $28(87.5)$ & & \\
\hline \multicolumn{5}{|l|}{ Tribe } \\
\hline Igbo & $33(17.5)$ & $156(82.5)$ & \multirow{2}{*}{0.174} & \multirow{2}{*}{0.677} \\
\hline Others (Hausa, Yoruba) & $4(4214.3)$ & $.24(85.7)$ & & \\
\hline \multicolumn{5}{|l|}{ Religion } \\
\hline Christianity & $36(17.6)$ & $168(82.4)$ & \multirow{2}{*}{0.856} & \multirow{2}{*}{0.355} \\
\hline Others (Muslim, traditional) & $1(7.7)$ & $12(92.3)$ & & \\
\hline \multicolumn{5}{|l|}{ Year of study } \\
\hline 200 level & $18(31.0)$ & 4069.0) & \multirow{5}{*}{15.699} & \multirow{5}{*}{0.003} \\
\hline 300 level & $5(20.8)$ & 19(79.2) & & \\
\hline 400 level & $10(14.3)$ & $60(85.7)$ & & \\
\hline 500 level & $2(323.6)$ & $53(96.4)$ & & \\
\hline 600 level & $2(20.0)$ & $8(80.0)$ & & \\
\hline \multicolumn{5}{|l|}{ Parent's relationship } \\
\hline Live together & $25(15.1)$ & $141(84.9)$ & \multirow{2}{*}{1.979} & \multirow{2}{*}{0.160} \\
\hline Others(single, separated, widow) & $12(23.5)$ & $39(76.5)$ & & \\
\hline \multicolumn{5}{|l|}{ Your parent's monthly income } \\
\hline$\leq 100$ & $9(15.8)$ & $48(84.2)$ & \multirow{3}{*}{0.663} & \multirow{3}{*}{0.718} \\
\hline $101-200$ & $9(18.8)$ & $39(81.3)$ & & \\
\hline$>200$ & $11(13.4)$ & $71(86.6)$ & & \\
\hline \multicolumn{5}{|l|}{ Your monthly allowance ranges } \\
\hline $1-10 \mathrm{k}$ & $10(15.9)$ & $53(84.1)$ & & \\
\hline $10-20 \mathrm{k}$ & $18(25.0)$ & $54(75.0)$ & 5605 & 0122 \\
\hline $21-30 k$ & $4(9.5)$ & $38(90.5)$ & 5.605 & 0.132 \\
\hline $31-40 \mathrm{k}$ & $4(11.1)$ & $24(88.9)$ & & \\
\hline Good personal interaction with yo & & & & \\
\hline Yes & $33(17.6)$ & $155(82.4)$ & 0251 & 0616 \\
\hline No & $4(13.8)$ & $25(86.2)$ & 0.251 & 0.010 \\
\hline Use of tobacco & & & & \\
\hline Use of tobacco & $0(0.0)$ & $13(100.0)$ & & \\
\hline Use of alcohol & $5(9.8)$ & $46(90.2)$ & 5.910 & 0.052 \\
\hline Use of caffeinated drinks/beverages & $19(21.3)$ & $70(78.7)$ & & \\
\hline
\end{tabular}


Table 6. Associations between factors and Panic Anxiety Disorder

\begin{tabular}{|c|c|c|c|c|}
\hline Variables & Anxiety n (\%) & No anxiety $n(\%)$ & Test statistic $\left(\chi^{2}\right)$ & p value \\
\hline \multicolumn{5}{|l|}{ Age(years) } \\
\hline$\leq 20$ & $5(10.0)$ & $45(90.0)$ & \multirow{4}{*}{4.240} & \multirow{4}{*}{0.237} \\
\hline $21-25 \mathrm{yrs}$ & $5(4.8)$ & $99(95.2)$ & & \\
\hline $26-30 \mathrm{yrs}$ & $1(2.1)$ & $46(97.9)$ & & \\
\hline Above $30 \mathrm{yrs}$ & $0(0.0)$ & $16(100.0)$ & & \\
\hline \multicolumn{5}{|l|}{ Sex } \\
\hline Male & $3(3.1)$ & 94(96.9) & \multirow{2}{*}{1.424} & \multirow{2}{*}{0.233} \\
\hline Female & $8(6.7)$ & 112(93.3) & & \\
\hline \multicolumn{5}{|l|}{ Marital status } \\
\hline Single & $11(5.9)$ & $174(94.1)$ & \multirow{2}{*}{2.004} & \multirow{2}{*}{0.157} \\
\hline Others (Married, Divorced, widow) & $0(0.0)$ & $32(100.0)$ & & \\
\hline \multicolumn{5}{|l|}{ Tribe } \\
\hline Igbo & $11(5.8)$ & $178(94.2)$ & \multirow{2}{*}{1.717} & \multirow{2}{*}{0.190} \\
\hline Others (Hausa, Yoruba) & $0(0.0)$ & $28(100.0)$ & & \\
\hline \multicolumn{5}{|l|}{ Religion } \\
\hline Christianity & $11(5.4)$ & 193(94.6) & \multirow{2}{*}{0.738} & \multirow{2}{*}{0.390} \\
\hline Others (Muslim, traditional) & $0(0.0)$ & $13(100.0)$ & & \\
\hline \multicolumn{5}{|l|}{ Year of study } \\
\hline 200 level & $6(10.3)$ & $52(89.7)$ & \multirow{5}{*}{$11^{\prime} 189$} & \multirow{5}{*}{0.025} \\
\hline 300 level & $0(0.0)$ & $24(100.0)$ & & \\
\hline 400 level & $2(2.9)$ & $68(97.1)$ & & \\
\hline 500 level & $1(1.8)$ & $54(98.2)$ & & \\
\hline 600 level & $2(20.0)$ & $8(80.0)$ & & \\
\hline \multicolumn{5}{|l|}{ Parent's relationship } \\
\hline Live together & $9(5.4)$ & 157(94.6) & \multirow{2}{*}{0.182} & \multirow{2}{*}{0.669} \\
\hline Others(single, separated, widow) & $2(3.9)$ & $49(96.1)$ & & \\
\hline \multicolumn{5}{|l|}{ Your parent's monthly income } \\
\hline$\leq 100$ & $2(3.5)$ & $55(96.5)$ & \multirow{3}{*}{2.512} & \multirow{3}{*}{0.285} \\
\hline $101-200$ & $5(10.4)$ & $43(89.6)$ & & \\
\hline$>200$ & $4(4.9)$ & $78(95.1)$ & & \\
\hline \multicolumn{5}{|l|}{ Your monthly allowance ranges } \\
\hline $1-10 \mathrm{k}$ & $3(4.8)$ & $60(95.2)$ & \multirow{4}{*}{0.456} & \multirow{4}{*}{0.928} \\
\hline $10-20 \mathrm{k}$ & $4(5.6)$ & $68(94.4)$ & & \\
\hline $21-30 \mathrm{k}$ & $3(7.1)$ & $39(92.9)$ & & \\
\hline $31-40 \mathrm{k}$ & $1(3.7)$ & $26(96.3)$ & & \\
\hline Good personal interaction with yo & & & & \\
\hline Yes & $11(5.9)$ & $177(94.1)$ & 1787 & 0181 \\
\hline No & $0(0.0)$ & $29(100.0)$ & 1.101 & 0.101 \\
\hline
\end{tabular}


Table 7. Associations between factors and Specific Phobia

\begin{tabular}{|c|c|c|c|c|}
\hline Variables & Anxiety $\mathrm{n}(\%)$ & No anxiety n (\%) & Test statistic $\left(\chi^{2}\right)$ & p value \\
\hline \multicolumn{5}{|l|}{ Age(years) } \\
\hline$\leq 20$ & $22(44.0)$ & $28(56.0)$ & \multirow{4}{*}{4.747} & \multirow{4}{*}{0.191} \\
\hline $21-25 \mathrm{yrs}$ & $28(26.9)$ & $76(73.1)$ & & \\
\hline $26-30 \mathrm{yrs}$ & $17(36.2)$ & $30(63.8)$ & & \\
\hline Above $30 \mathrm{yrs}$ & $6(37.5)$ & $10(62.5)$ & & \\
\hline \multicolumn{5}{|l|}{ Sex } \\
\hline Male & $28(28.9)$ & $69(71.1)$ & \multirow{2}{*}{1.791} & \multirow{2}{*}{0.181} \\
\hline Female & $45(37.5)$ & $75(62.5)$ & & \\
\hline \multicolumn{5}{|l|}{ Marital status } \\
\hline Single & $55(29.7)$ & $130(70.3)$ & \multirow{2}{*}{8.595} & \multirow{2}{*}{0.003} \\
\hline Others (Married, Divorced, widow) & $18(56.3)$ & $14(43.8)$ & & \\
\hline \multicolumn{5}{|l|}{ Tribe } \\
\hline Igbo & $60(31.7)$ & $129(68.3)$ & \multirow{2}{*}{2.355} & \multirow{2}{*}{0.125} \\
\hline Others (Hausa, Yoruba) & $13(46.6)$ & $.15(53.6)$ & & \\
\hline \multicolumn{5}{|l|}{ Religion } \\
\hline Christianity & $67(32.8)$ & $137(67.2)$ & \multirow{2}{*}{0.970} & \multirow{2}{*}{0.325} \\
\hline Others (Muslim, traditional) & $6(46.2)$ & $.7(53.8)$ & & \\
\hline \multicolumn{5}{|l|}{ Year of study } \\
\hline 200 level & $21(36.2)$ & $37(63.8)$ & \multirow{5}{*}{2.808} & \multirow{5}{*}{0.590} \\
\hline 300 level & $8(33.3)$ & $16(66.7)$ & & \\
\hline 400 level & $20(28.6)$ & $50(71.4)$ & & \\
\hline 500 level & $22(40.0)$ & $33(60.0)$ & & \\
\hline 600 level & $2(20.0)$ & $8(80.0)$ & & \\
\hline \multicolumn{5}{|l|}{ Parent's relationship } \\
\hline Live together & $55(33.1)$ & $111(66.9)$ & \multirow{2}{*}{0.082} & \multirow{2}{*}{0.775} \\
\hline Others(single, separated, widow) & $18(35.3)$ & $33(64.7)$ & & \\
\hline \multicolumn{5}{|l|}{ Your parent's monthly income } \\
\hline$\leq 100$ & $25(43.9)$ & $32(56.1)$ & \multirow{3}{*}{7.603} & \multirow{3}{*}{0.022} \\
\hline $101-200$ & $21(43.8)$ & $27(56.3)$ & & \\
\hline$>200$ & $20(24.4)$ & $62(75.6)$ & & \\
\hline \multicolumn{5}{|l|}{ Your monthly allowance ranges } \\
\hline $1-10 \mathrm{k}$ & $32(50.8)$ & $31(49.2)$ & & \\
\hline $10-20 \mathrm{k}$ & $20(27.8)$ & $52(72.2)$ & 14.929 & 0.002 \\
\hline $21-30 \mathrm{k}$ & $7(16.7)$ & $35(83.3)$ & & \\
\hline $31-40 \mathrm{k}$ & $10(37.0)$ & $17(63.0)$ & & \\
\hline Good personal interaction with yo & & & & \\
\hline Yes & $62(33.0)$ & $126(67.0)$ & & \\
\hline No & $11(37.9)$ & $18(62.1)$ & 0.276 & 0.599 \\
\hline Use of tobacco & & & & \\
\hline Use of tobacco & $6(46.2)$ & $7(53.8)$ & & \\
\hline Use of alcohol & $22(43.1)$ & $29(56.9)$ & 3.486 & 0.175 \\
\hline Use of caffeinated drinks/beverages & $26(29.2)$ & $63(70.8)$ & & \\
\hline
\end{tabular}


Table 8. Associations between factors and Obsessive-compulsive disorder

\begin{tabular}{|c|c|c|c|c|}
\hline Variables & Anxiety n (\%) & No anxiety $n(\%)$ & Test statistic $\left(\chi^{2}\right)$ & p value \\
\hline \multicolumn{5}{|l|}{ Age(years) } \\
\hline$\leq 20$ & $11(22.0)$ & $39(78.0)$ & \multirow{4}{*}{7.574} & \multirow{4}{*}{0.056} \\
\hline $21-25 y r s$ & $18(17.3)$ & $86(82.7)$ & & \\
\hline $26-30 y r s$ & $4(8.5)$ & $43(91.5)$ & & \\
\hline Above $30 y r s$ & $6(37.5)$ & $10(62.5)$ & & \\
\hline \multicolumn{5}{|l|}{$\operatorname{Sex}$} \\
\hline Male & $13(13.4)$ & $84(86.6)$ & \multirow{2}{*}{2.485} & \multirow{2}{*}{0.115} \\
\hline Female & $26(21.7)$ & $94(78.3)$ & & \\
\hline \multicolumn{5}{|l|}{ Marital status } \\
\hline Single & $29(15.7)$ & $156(84.3)$ & \multirow{2}{*}{4.489} & \multirow{2}{*}{0.034} \\
\hline Others (Married, Divorced, widow) & $10(31.3)$ & $22(68.8)$ & & \\
\hline \multicolumn{5}{|l|}{ Tribe } \\
\hline Igbo & $32(16.9)$ & $157(83.1)$ & \multirow{2}{*}{1.077} & \multirow{2}{*}{0.299} \\
\hline Others (Hausa, Yoruba) & $7(25.0)$ & $21(75.0)$ & & \\
\hline \multicolumn{5}{|l|}{ Religion } \\
\hline Christianity & $36(17.6)$ & $168(82.4)$ & \multirow{2}{*}{0.244} & \multirow{2}{*}{0.621} \\
\hline Others (Muslim, traditional) & $3(23.1)$ & $10(76.9)$ & & \\
\hline \multicolumn{5}{|l|}{ Year of study } \\
\hline 200 level & $18(31.0)$ & $40(69.0)$ & \multirow{5}{*}{10.918} & \multirow{5}{*}{0.028} \\
\hline 300 level & $5(20.8)$ & $19(79.2)$ & & \\
\hline 400 level & $7(10.0)$ & $63(90.0)$ & & \\
\hline 500 level & $7(12.7)$ & $48(87.3)$ & & \\
\hline 600 level & $2(20.0)$ & $8(80.0)$ & & \\
\hline \multicolumn{5}{|l|}{ Parent's relationship } \\
\hline Live together & $31(18.7)$ & $135(81.3)$ & \multirow{2}{*}{0.236} & \multirow{2}{*}{0.627} \\
\hline Others(single, separated, widow) & $8(15.7)$ & $43(84.3)$ & & \\
\hline \multicolumn{5}{|l|}{ Your parent's monthly income } \\
\hline$\leq 100$ & $8(14.0)$ & $49(86.0)$ & \multirow{3}{*}{0.748} & \multirow{3}{*}{0.688} \\
\hline $101-200$ & $9(18.8)$ & $39(81.3)$ & & \\
\hline$>200$ & $16(19.5)$ & $66(80.5)$ & & \\
\hline \multicolumn{5}{|l|}{ Your monthly allowance ranges } \\
\hline $1-10 \mathrm{k}$ & $13(20.6)$ & $50(79.4)$ & & \\
\hline $10-20 \mathrm{k}$ & $16(22.2)$ & $56(77.8)$ & 4752 & 0101 \\
\hline $21-30 \mathrm{k}$ & $8(19.0)$ & $34(81.0)$ & 4.135 & 0.191 \\
\hline $31-40 \mathrm{k}$ & $1(3.7)$ & $26(96.3)$ & & \\
\hline Good personal interaction with yo & & & & \\
\hline Yes & $32(17.0)$ & $156(83.0)$ & 0962 & 0252 \\
\hline No & $7(24.1)$ & $22(75.9)$ & 0.005 & 0.535 \\
\hline Use of tobacco & & & & \\
\hline Use of tobacco & $3(23.1)$ & $10(76.9)$ & & \\
\hline Use of alcohol & $6(11.8)$ & $45(88.2)$ & 3.925 & 0.141 \\
\hline Use of caffeinated drinks/beverages & $23(25.8)$ & $66(74.2)$ & & \\
\hline
\end{tabular}




\section{Discussion}

In the present study, the overall prevalence of anxiety disorders among medical students was $14.3 \%$. Specifically, the relative frequencies of GAD, PTSD, SAD, panic disorder, specific disorder and OCDs were $39.6 \%, 32.3 \%$, $17.1 \%, 5.1 \%, 33.6 \%$ and $18.0 \%$ respectively. The overall prevalence of anxiety disorders noted in this study is comparatively lower than the prevalence of $47 \%$ reported among medical students with multiple nationalities in Saudi Arabia: comprising Arabs, South Asians, and North Americans (Bibi \& Nasir Ali, 2015). Interestingly, the study observed a higher prevalence of $63 \%$ among these students prior to examinations which rapidly declined to $47 \%$ after the examinations. Elsewhere in Malaysia, Sherina et al (2005) also noted a higher prevalence of 38.4\% in their study among Malaysian medical students. Disparities between these prevalence rates may be due to study methodology, sample size, the type of questionnaire used and ethno-geographic variables. Nevertheless, the high prevalence rates of anxiety disorders among medical students underscores the need to possibly review the curriculum, examination methods, study environment and accommodation, which are all potential stressors and triggers of anxiety.

We noted in this study that GAD was significantly associated with gender and year of study. Specifically, there was a female preponderance among medical students in the clinical setting (especially $4^{\text {th }}$ and $5^{\text {th }}$ year of study) who exhibited this disorder, which was however least observed among the final year students. Challenges of facing new learning techniques in the clinical class such as clerking of and interacting with patients, as well as exposure to hospital environment to which these students were not hitherto exposed are all contributory factors. For the final year students, expectations to become competent doctors and to acquire good academic results may account for the low prevalence of the disorder among them (Zaid et al., 2007).

It is generally believed that females tend to have more anxiety symptoms than their male counterparts: an assumption which has been corroborated by the finding of the current study. Suggested reasons for this tendency include greater social freedom for expression of feelings, physiologic and neuro-hormonal factors ((Blanch et al., 2008; Andrade et al., 2006; Soumya et al., 2006). The prevalence rate of $32.4 \%$ for PTSD obtained in the current study is less than the rates of $21.9 \%$ and $22.9 \%$ reported by Shrestha et al (2015), and Ameel et al (2011) respectively. Again, differences in study location, the questionnaire used as screening tool for anxiety disorders and sample size may account for this disparity in prevalence rates. Interestingly, PTSD were mainly noticed in the second year of study among medical students evaluated in the current study: a period these students are exposed to cadaveric dissection in learning the subject of Anatomy. A study by Peter \& Lawrence (1990) noted that approximately $5 \%$ of the students in Anatomy class reported nightmares, intrusive visual images, insomnia, depression, and learning impairments. These psychologic responses strongly resemble PTSD: making the authors to conclude that the Anatomy laboratory may represent a significant emotional challenge to many medical students, as it may represent the student's first intimate experience with illness and death (Peter \& Lawrence, 1990). Furthermore, the non-significant association of PTSD with age, marital status, profession, previous disaster experience, tragic events with relatives was also corroborated by Shrestha (2015).

The prevalence rate of $33.6 \%$ for SAD reported in this study is lower than the $56 \%$ recorded in Malaysia by Gill \& Mohammad (2010). Again, SAD and panic disorder appeared significantly related to the year of study. For instance, these disorders were worse among 200 level students compared to other levels of medical training. Importantly, the reason for this observation may be the commencement of exposure of the students at this level of study to morbidity and mortality stereotypes like cadaveric dissection.

The present study also found that medical students who were not married were more likely to have phobia compared to their married counterparts. In addition, students whose parents were low-income earners and students with low monthly allowance all showed more tendency to phobia compared to students whose parents were high-income earners and their counterparts with high monthly allowance. Although Ali (2013) reported no significant difference in the prevalence of social phobia with respect to gender, family income or type of residence, another author in Baghdad, Iraq (Abdul, 2012) noted a significant association of phobia among medical students with rural residence and low socio-economic background. This study shows a high tendency for married students and those from high socio-economic class to have some protection from the component anxiety disorders. Although the reason remains unclear, it has been documented that married students were significantly less susceptible to anxiety disorders compared to their single-status colleagues while those not involved in a romantic relationship were found to have significantly more anxiety disorders that those who were not (Johari \& Hashim, 2009).

The prevalence of $33.6 \%$ for specific phobia obtained in this study compares well with the prevalence of $27 \%$ among medical students in the study by Ali (2013). Remarkably, it is much higher than the rates among 
non-medical undergraduate students by reported Johari \& Hashim (2009), who documented a life-time and 12 -month prevalence of $9.4 \%$ and $8.5 \%$ respectively. The reason for this high prevalence rate of phobia among medical students when compared to their non-medical counterparts may include fear of examination failure and course-withdrawal associated with medical schools.

Finally, in the present study, OCDs were found to be significantly related to marital status and year of study. These findings are in tandem with the observations of Torres et al (2016), who noted that prevalence rates for this disorder were higher among fresher students (year 1 and 2 students). According to these authors, the 'obsession' dimension was also associated with being a 'freshman' (Torres et al., 2016).

\subsection{Study Limitations}

The study was a single-center evaluation of medical students. Findings may not therefore be representative of these disorders among medical students in Nigeria. Secondly, the standardized questionnaires used as screening tools were self-administered and not interviewer-administered, giving room for possible wrong responses among students who might fully not have comprehended the items.

\section{Conclusion}

Medical students in Nigeria are prone to a spectrum of anxiety disorders. This susceptibility is influenced by socio-demographic characteristics.

\section{Acknowledgements}

We acknowledge Onyiaorah C, Udeh C and Ugwu C who helped immensely in the data collection.

\section{Funding}

No external funding sources for the study

\section{Authors' Contribution}

All authors contributed to the conception and writing of the manuscript. AEC analyzed the manuscript.

\section{Disclosure}

The authors hereby declare that there are no conflicts of interests.

\section{References}

Abdul, Z. M. (2012). Social phobia among Al Qadissya medical student: prevalence, Academic performance and response to different treatments. J Fac Med Baghdad, 54, 33-7.

Ali, K. S. (2013). Social phobia among medical students. Eur Psychiatry, 28, 1 https://doi.org/10.1016/S0924-9338 (13)76032-2

Ameel, F. A., Nesif, J. A., Jawad, K. A., \& Diar, H. T. (2011). Post-Traumatic Stress Disorder among University Students in Baghdad: A Preliminary Report. Iraqi J Comm Med, 24, 287-90. Retrieved from https://www.iasj.net/iasj?func $=$ fulltext\&aId $=61173$

Andrade, L. H., Viana, M. C., \& Silveira, C. M. (2006). Epidemiology of women's psychiatric disorders. Rev Psiq Clin, 33, 43-54. http://dx.doi.org/10.1590/S0101-60832006000200003

Asaad, Q. Al-Y., \& Aqeel, I. S. (2010). Prevalence of post-traumatic stress disorder among Basra medical students. The Medical Journal of Basra University, MJBU, 28, 62-6.

Bibi, K., \& Nasir, A. A. (2015). Stress, anxiety, and depression among medical students in a multiethnic setting. Neuropsychiatr Dis Treat, 11, 1713-22. https://doi.org/10.2147/NDT.S83577

Blanch, D. C., Hall, J. A., Roter, D. L., \& Frankel, R. M. (2008). Medical student gender and issues of confidence. Pat Educ Counsel, 72, 374-81. https://doi.org/10.1016/j.pec.2008.05.021

Bostanci, M., Ozdel, O., Oguzhanoglu, N. K., Ozdel, L., \& Ergin, A. (2005) Depressive symptomatology among university students in Denizli, Turkey: prevalence and sociodemographic correlates. Croat Med, 46, 96-100. Retrieved from https://www.ncbi.nlm.nih.gov/pubmed/15726682

Connor, K. M., Kobak, K. A., Churchill, L. E., Katzelnick, D., \& Davidson, J. R. (2001). Mini-SPIN: A brief screening assessment for generalized social anxiety disorder. Depress Anxiety, 14, 137-40. https://doi.org/10.1002/da.1055

Eller, T., Aluoja, A., Vasar, V., \& Veldi, M. (2006). Symptoms of anxiety and depression in Estonian medical students with sleep problems. Depress Anxiety, 23, 250-6. https://doi.org/10.1002/da.20166 
Gelder, M., Paul, H., \& Philip, C. (2006). Shorter Oxford Textbook of Psychiatry (5th Ed.). Oxford University press. Retrieved from https://global.oup.com/academic/product/shorter-oxford-textbook-ofpsychiatry-9780198747437

Gill, J., \& Mohammad, S (2010). Social anxiety among medical undergraduate students in Malaysia. Eur Psychiatry, 25, 347. https://doi.org/10.1016/S0924-9338 (10)70344-8

Goodwin, R. D., Faravelli, C., Rosi, S., Cosci, F., Truglia, E., de Graaf, R., \& Wittchen, H. U. (2005). The epidemiology of panic disorder and agoraphobia in Europe. European Neuropsychopharmacology, 15(4), 435-443. https://doi.org/10.1016/j.euroneuro.2005.04.006

Johari, A. B., \& Hashim, I. N. (2009). Stress and coping strategies among medical students in National University of Malaysia, Malaysia University of Sabah and University Kuala Lumpur Royal College of Medicine Perak. $J$ Com Health, 15, 106-15. https://onlinelibrary.wiley.com/doi/abs/10.1002/ca.980030308

Karno, M., Golding, J. M., Sorenson, S. B., \& Burnam, M. A. (1988). The epidemiology of obsessive compulsive disorder in five US communities. Arch Gen Psychiatry, 45, 1094-9. https://doi.org/10.1038/mp.2008.94

Kessler, R. C., Chiu, W. T., Demler, O., Merikangas, K. R., \& Walters, E. E. (2005). Prevalence, severity, and co-morbidity of 12-month DSM-IV disorders in the National Co-morbidity Survey Replication. Arch Gen Psychiatry, 62, 617. https://doi.org/10.1001/archpsyc.62.6.617

Laidlaw, A. H. (2009). Social anxiety in medical students: Implications for communication skills teaching. Med Teach, 31, 649-54. https://doi.org/10.1080/01421590802208867

Leon, A. C., Olfson, M., Broadhead, W. E., Barrett, J. E., Blacklow, R. S., Keller, M. B., ... \& Weissman, M. M. (1995). Prevalence of mental disorders in primary care: implications for screening. Archives of Family Medicine, 4(10), 857.https://doi.org/10.1001/archfami.4.10.857

Margot, S. (2004). "Social anxiety disorder- beyond shyness" (PDF). How healthy are Canadians? Statistics Canada Annual Report, 15, 58. Retrieved from https://www.statcan.gc.ca/pub/82-003-s/2004000/4148954-eng.htm

Merikangas, K. R. (2004). Clinical Features of Anxiety Disorders. Comprehensive Textbook of Psychiatry. In: Kaplan HI, Sadock BJ (Eds), Lippincott Williams \& Wilkins, 1104-26. 12. Retrieved from https://www.amazon.com/Kaplan-Sadocks-Comprehensive-Textbook-Psychiatry/dp/0781768993

Oyedeji, G. A. (1985). Socio-economic and cultural background of hospitalized children in Ilesha. Nig J Paediatr, $12,111-7$

Peter, F., \& Lawrence, H (1990). Post-traumatic stress among medical students in the anatomy dissection laboratory. Clin Anatomy, 3, 219-26. https://doi.org/10.1002/ca.980030308

Robert, L. S., Kurt, K., Janet, B. W., \& Bernd, L. (2006). The GAD-7. Arch Intern Med, 166, 1092-1097. https://doi.org/10.1001/archinte.166.10.1092

Sherina, M. S., Rampal, L., \& Kaneson, N. (2005). The prevalence of emotional disorders among medical students. Malaysian J Med Health Sci, 1, 1320-27. https://doi.org/10.1111/j.1444-1683.2003.00089.x

Shrestha, R. (2015). Post-traumatic Stress Disorder among Medical Personnel after Nepal earthquake. J Nepal Health Res Counc, 13, 144-8. https://www.ncbi.nlm.nih.gov/pubmed/26744200

Soumya, S., Richa, T., Pankaj, V., Rohit, K., \& Sarthak, S. (2015). Prevalence of anxiety and insomnia among the medical students: A cross sectional study at a tertiary care hospital. Int J Basic Applied Med Sci, 5, 45-50. Retrieved from http://www.cibtech.org/J-MEDICAL-SCIENCES/PUBLICATIONS/2015/Vol_5 No_2/09-JMS-009-SOUMYA-PREVALENCE.pdf

Stein, Murray, B., \& Gorman, J. M. (2001). Unmasking social anxiety disorder. J Psychiatr Neuroscience, 26, 185-9

Tabalipa, F. D. O., Souza, M. F. D., Pfützenreuter, G., Lima, V. C., Traebert, E., \& Traebert, J. (2015). Prevalence of anxiety and depression among medical students. Revista Brasileira De Educação Médica, 39(3), 388-394. http://dx.doi.org/10.1590/1981-52712015v39n3e02662014

Torres, A. R., Cruz, B. L., Vicentini, H. C., Lima, M. C., \& Ramos-Cerqueira, A. T. (2016). Obsessive-Compulsive Symptoms in Medical Students: Prevalence, Severity, and Correlates. Acad Psychiatry, 40, 46-54. https://doi.org/10.1007/s40596-015-0357-2

The Primary Care PTSD Screen-Mental Health. Retrieved 1st August, 2016, from 
http://www.mentalhealth.va.gov/community...ders/docs/PCPTSD_Screen.pd

Wittchen, H. U. (2002). Generalized anxiety disorder: prevalence, burden, and cost to society. Depress Anxiety, 16, 162-71. https://doi.org/e 10.1002/da.10065

Zaid, Z. A., Chan, S. C., \& Ho, J. J. (2007). Emotional disorders among medical students in a Malaysian private medical school. Singapore Med J, 48, 895-9. Retrieved from https:/www.ncbi.nlm.nih.gov/pubmed/17909672

\section{Copyrights}

Copyright for this article is retained by the author(s), with first publication rights granted to the journal.

This is an open-access article distributed under the terms and conditions of the Creative Commons Attribution license (http://creativecommons.org/licenses/by/4.0/). 